\title{
Editorial
}

\section{The Headache and adverse events due to the use of personal protective equipment to combat Covid-19}

\author{
Erlene Roberta Ribeiro dos Santos
}

Department of Collective Health at the Academic Center of the Federal University of Pernambuco, Vitória de Santo Antão, Pernambuco, Brazil.

\section{$\triangle$}

erlene.santos@ufpe.br

Edited by

Marcelo Moraes Valença
Headache deserves specific attention in the context of the current health situation when it can be potentially associated with the use of three specific artifacts, during the Covid-19 pandemic, in which it is necessary to change behavioral habits across the planet. Personal protective equipment such as a mask, face shield, and glasses for healthcare professionals has never been more widespread. Personal protective equipment is already routinely used in healthcare environments such as urgent and emergency services, laboratories, clinics, and hospitals. However, in the context of the Covid-19 pandemic, the use for the protection of health professionals in the fight against the coronavirus has been occurring for a long time for hours during work shifts.' These devices compress the scalp tissue circumferentially and often leave pressure marks after its removal, as is often observed in the case of the mask.

This situation has affected the work environment of professionals who have been at the forefront of combating Covid-19 since December 2019, in units dedicated to the care of infected patients. Therefore, we wonder about the need and importance of exploring the adverse event of prolonged use of personal protective equipment such as mask, face shield, and goggles associated with the triggering of external pressure headaches.

Personal protective equipment has fundamental importance to avoid contagion in the treatment of patients infected with the coronavirus. However, the use of caps, masks, face shields, eye protection glasses, and other accessories necessary to comply with the clinical protocols defined for patient care has been drawing attention to an important issue of occupational health, as mandatory and uninterrupted times for hours of the work shift can generate significant compression ${ }^{2}$ in the innervated tissue of the head and physical changes.

A worldwide study carried out via web3 ${ }^{3}$, in April 2020, reveals a description of adverse effects on the use of protective equipment associated with the period of more than 4 uninterrupted hours carried out with professionals working in intensive care units. Of the 2,711 responses to the questionnaire submitted online, 1,797 (67\%) doctors, $744(27 \%)$ nurses, and $170(6 \%)$ paramedics were identified. The majority $(1,557 ; 58 \%)$ reported the routine use of mask type FFP2 or N95, long-sleeved waterproof aprons (1,623; 67\%), and face shields (1,564; 62\%). The adverse effects were associated with variables such as the duration of use in the longest shift, feeling of discomfort due to heat, sensitivity in areas of pressure, headache, and extreme exhaustion.

The etiology of this type of headache is triggered by external pressure resulting from the sustained compression of the soft tissues of the epicrania, associated with the use of the equipment on the head ${ }^{2}$, which can lead to work disability, direct costs mainly related to absenteeism, indirect costs often linked to the use of medications, both individual and health systems. Also, other elements such as the high level of exposure to stressors, such as the news of deaths by Covid-19 that occurred with patients and coworkers, can increase the frequency of crises and illness processes associated with other comorbidities., 4 
A recent study' presents evidence about the N95 face mask that efficiently protects against respiratory droplets. On the other hand, it highlights evidence showing adverse effects such as excoriation on the bridge of the nose, which can be caused by the excessive pressure and hardness of the equipment's metal clip, causing a painful sensation and post-use marks for long periods on the facial tissues., 5

Another previous study measured the impact of headaches associated with prolonged use of N95 masks as a risk factor, with 217 health workers, revealing that $79 / 217(37.3 \%)$ reported headache associated with the use of N95 mask, 26/217 (32.9\%) reported headache frequency more than six times a month, $7.6 \%$ requested sick leave due to work disability (ranging from 1 to 4 days, with an average of two days), $47 / 217$ (59.5\%) resorted to crisis abortion medication and $4 / 2,017$ used preventive medication during the period of use of the equipment, showing positive statistical significance for the association of pre-existing headaches and continuous use of the N95 face mask. ${ }^{6}$

The pressure exerted by the facial protector, depending on the elastic adjustment, can pressure the region of the epicranial muscles ${ }^{5}$, which are areas of hypersensitivity and can reproduce pain symptoms, which can also enhance the headache characteristics. The headaches that occur in professionals who use masks, face shield, and goggles for eye protection may arise due to the pressure of the strap in the neck or the occipital area, on the superficial nerves, which may aggravate an underlying cervical tension and potentiate the headache associated with the use of the three equipment combined simultaneously or alternating. ${ }^{6}$

For those who already suffer from primary headaches such as migraine, the damage can be greater, as the continued use of the accessory by pressing on sensitive areas for an extended period can increase the chance of triggering a crisis.

It is feasible to point out that there are few studies on headaches due to external pressure from the use of a band surrounding the head region, hat ${ }^{7}$, helmets ${ }^{8}$ and tight swimming goggles that are pointed out as the cause of constant and intense pain in the area pressed by the object. This evidence already points to the importance of headache potentially triggered by the use of adornments that generate pressure in the pericranial tissue. ${ }^{7}$

Because of the complex current scenario with efforts aimed at combating Covid-19, this brief article warns of the need for more in-depth research on the adverse events potentially triggered by the three most used personal protective equipment in health services (mask, face shield and eye protection glasses), as daily and continuous exposure for prolonged periods by professionals can bring consequences.?

Based on these notes, it is recommended that greater attention be paid to the care with the improvement of protective equipment as an object of study, in the search for alternatives that can minimize the damage caused by prolonged use for the occupational health of the area worker.

Erlene Roberta Ribeiro dos Santos

https://orcid.org/0000-0003-3334-3408

\section{References}

1. Hu K, Fan J, Li X, Gou X, Li X, Zhou X. The adverse skin reactions of health care workers using personal protective equipment for COVID-19. Medicine (Baltimore). 2020;99:e20603.

2. Headache Classification Committee of the International Headache Society (IHS) The International Classification of Headache Disorders, 3rd edition. 2018;38:1-211.

3. Tabah A, Ramanan M, Laupland KB, et al. Personal protective equipment and intensive care unit healthcare worker safety in the COVID-19 era (PPE-SAFE): An international survey. Journal of critical care. 2020;59:70-75.

4. Polk AN, Protti TA, Smitherman TA. Allodynia and Disability in Migraine: The Mediating Role of Stress. Headache. 2020;60:2281-2290.

5. Alonso-Blanco C, Fernández-de-las-Peñas C, Fernández-Mayoralas DM, de-la-Llave-Rincón Al, Pareja JA, Svensson P. Prevalence and anatomical localization of muscle referred pain from active trigger points in head and neck musculature in adults and children with chronic tension-type headache. Pain medicine (Malden, Mass). 2011;12:1453-1463.

6. Lim ECH, Seet RCS, Lee K-H, Wilder-Smith EPV, Chuah BYS, Ong BKC. Headaches and the N95 face-mask amongst healthcare providers. Acta Neurologica Scandinavica. 2006;113:199-202.

7. Agarwal A, Agarwal S, Motiani P. Difficulties Encountered While Using PPE Kits and How to Overcome Them: An Indian Perspective. Cureus. 2020;12:e11652.

8. Rahmani Z, Kochanek A, Astrup JJ, Poulsen JN, Gazerani P. Helmet-induced headache among Danish military personnel. Scandinavian Journal of Public Health. 2017;45:818-823.

9. Krymchantowski AV. Headaches Due to External Compression. Current Pain and Headache Reports. 2010;14:321-324. 